\title{
An Egyptian child with erythromelalgia responding to a new line of treatment: a case report and review of the literature
}

\author{
Samir M Al-Minshawy and Abdel-Azeem M El-Mazary*
}

\begin{abstract}
Introduction: Erythromelalgia is a rare clinical syndrome characterized by episodic erythema, warmth and intense burning pain, which commonly involves the extremities. For those affected, this disorder may lead to significant long-term morbidity. Unfortunately, to date, no definitive therapy is available. This case report describes an Egyptian child with primary erythromelalgia that manifested at an early age and showed partial response to therapy with cetirizine hydrochloride. This anecdotal case report may have a diagnostic value for clinicians who have not seen this disorder.
\end{abstract}

Case presentation: A 34-month-old previously healthy right-handed Hamitic boy without any significant past medical history presented at the age of 2 years with episodic bilateral pain in his feet. His mother reported associated warmth and erythema localized to his feet that never extended beyond his ankle joints. This pain is triggered by exertion and/ or warm temperature exposure and is relieved by cooling measures. The diagnosis of erythromelalgia was made based on the patient's medical history and a thorough physical examination during the episodes. No evidence of local or systemic infection was present. Other causes for the symptoms were excluded by a negative extensive diagnostic work-up. Our patient did not respond to ibuprofen $(15 \mathrm{mg} / \mathrm{kg} / \mathrm{dose})$ three times a day but partial improvement with the oral non-sedating antihistaminic cetirizine hydrochloride $(2.5 \mathrm{mg} / \mathrm{kg} /$ once daily) was observed. When the child stopped cetirizine hydrochloride for 1 month as a test, the symptoms became aggravated and were relieved when cetirizine therapy was restarted. Cetirizine hydrochloride had not previously been reported to have this effect in children with erythromelalgia.

Conclusions: Erythromelalgia is a clinical syndrome of which the etiology, diagnosis and management are controversial. We describe a case of a 34-month-old Egyptian child with primary erythromelalgia that manifested at an early age. We believe that this is the first Egyptian case report of this kind in the literature. Partial response of this patient to cetirizine hydrochloride may grant us a new clue to understanding this mysterious condition.

Keywords: Cetirizine hydrochloride, Egyptian children, Erythromelalgia

\section{Introduction}

Erythromelalgia (EM) is a rare disorder in children characterized by episodic erythema, warming and burning pain, which commonly involves the extremities [1]. EM can be primary (which may be sporadic or familial) or secondary to other causes including but not limited to autoimmune disorders, myeloproliferative and/or neuropathic conditions [2] (Table 1).

\footnotetext{
* Correspondence: abdelazeemhemed@yahoo.com

Department of Pediatrics, Minia University, Postcode 61111 Minia city, Egypt
}

Symptoms are triggered by physical exertion and/or a warm environment and can be relieved by cooling. Episodes may last from minutes to hours. Early recognition of EM is important but difficult due to the rare nature of the disorder [3].

\section{Case presentation}

Our patient was a 34-month-old right-handed Hamitic boy who presented with insidious intermittent attacks of bilateral intense pain, warmth and flushing of feet, each lasting minutes to hours. The age of onset for these symptoms was 2 years. His symptoms had the tendency

\section{() BioMed Central}


Table 1 Reported causes of secondary erythromelalgia [2]

\begin{tabular}{ll}
\hline $\begin{array}{l}\text { Myeloproliferative diseases } \\
\text { and blood disorders }\end{array}$ & Drugs \\
Essential thrombocythemia & Cyclosporine \\
Polycythemia vera & Verapamil \\
Myelodysplastic syndrome & Nicardipine \\
Pernicious anemia & Nifedipine \\
Thrombotic and immunologic & Norephedrine \\
thrombocytopenic purpuras & Bromocriptine and pergolide \\
Infectious diseases & Connective tissue diseases \\
Human immunodeficiency virus & Systemic lupus erythematosus \\
Hepatitis B vaccine & Vasculitis \\
Influenza vaccine & Neoplastic \\
Infectious mononucleosis & Paraneoplastic syndrome \\
Pox virus & Astrocytoma \\
Neuropathic & Malignant thymoma \\
Diabetic neuropathy & Others \\
Peripheral neuropathies & Mushroom ingestion \\
Neurofibromatosis & Mercury poisoning \\
Riley-day syndrome & \\
Multiple sclerosis & \\
\hline
\end{tabular}

to be symmetrical, localized to his feet and never extended proximally beyond his ankle joints; they were precipitated and worsened with exercise and/or warm temperature exposure such as covering his legs with blankets and were abated by cooling measures like cold water. There was no history of similar conditions in his family or drug intake before the precipitation of the attacks. He had no history of previous blood transfusion. He looks well, with no manifestations of acute illness. Physical examinations during multiple visits revealed: normal vital signs; no pallor, jaundice or cyanosis were present; no organomegaly or lymphadenopathies were present; only both his feet appeared red in color (Figure 1) and warm. Extensive investigations were done for exclusion of other diseases causing pain and/or flushing of both lower limbs as well as for exclusion of secondary EM.

In this child, the investigations revealed normal complete blood count $(\mathrm{CBC})$ with differential, normal serum immunoglobulin E (IgE) titre $(11 \mathrm{IU} / \mathrm{mL})$, normal levels of serum cholesterol $(146 \mathrm{mg} / \mathrm{dL})$ and triglycerides $(49 \mathrm{mg} / \mathrm{dL})$, normal levels of serum urea $(16 \mathrm{mg} / \mathrm{dL})$ and serum creatinine $(0.7 \mathrm{mg} / \mathrm{dL})$, normal liver enzymes (alanine aminotransferase $22 \mathrm{U} / \mathrm{L}$ and aspartate aminotransferase 34U/L), normal serum uric acid $(3.7 \mathrm{mg} / \mathrm{dL})$, negative antistreptolysin $\mathrm{O}$ titre, and normal urine analysis and stool analysis. The fasting and 2 hours post-prandial blood glucose levels were 89 and $122 \mathrm{mg} / \mathrm{dL}$ respectively.

In addition, the results of an X-ray of the bones in both his feet and legs and a Doppler of the arteries of both his lower limbs were normal, normal nerve conduction velocities of both peroneal nerves and normal bone marrow biopsy were present. A pelvic and abdominal sonography and brain computed tomography (CT) were done and all were normal.

A skin biopsy was performed, showing nonspecific changes consistent with the diagnosis of primary EM (Figure 2) in the form of numerous telangiectatic blood vessels in the capillary dermis associated with sparse perivascular mononuclear cell infiltrate and some vessels showed swelling of the endothelial lining. The intimal thickening and thrombi seen in secondary EM were lacking.

He received ibuprofen $(15 \mathrm{mg} / \mathrm{kg} /$ dose three times a day) for 2 to 3 weeks but no relief of his symptoms was observed, but he did report a partial response to cetirizine hydrochloride $(2.5 \mathrm{mg} / \mathrm{kg} /$ once daily). When the child stopped cetirizine hydrochloride for 1 month as a test, his symptoms became aggravated but were relieved when cetirizine therapy was restarted; the frequency and severity of the attacks were reduced. His mother was advised that he should avoid all conditions that exacerbated his symptoms and that she should expose his lower limbs to cold air (fan) during an attack and avoid his exposure to cold or ice water to avoid ischemia of both his lower limbs. She was advised on the benefit of routine follow-up evaluation in the pediatric clinic for follow-up by $\mathrm{CBC}$ and, if necessary, any other investigations needed. Finally, the diagnosis of primary EM was made based on the clinical history and examination.

\section{Discussion}

In 1878, Mitchell [4] described and named 'erythromelalgia'; the name 'erythromelalgia' reflected the characteristic findings of redness (erythros) and pain (algos) involving the extremities (melos). Unfortunately, there is at present no confirmatory diagnostic test and the diagnosis is based on taking a careful history and physical examination during the episodes. With the advent of digital photography, photographs can be very helpful to document the events of erythema. Thermography can reveal the increased skin temperature in the affected area, but this is not necessary to establish the diagnosis $[2,3]$.

The incidence and prevalence of EM are difficult to calculate due to the patient's failure to recognize the condition when the symptoms are mild, and physicians may fail to make the diagnosis because this is a rare and relatively unknown disorder. The proposed incidence is 2.5 to 3.3 per million per year [5], with a prevalence of 18 to 20 per million in the Norwegian population [6]. A recent retrospective study in Sweden reported an incidence of 0.36 cases per 100,000 population [7]. The incidence and prevalence of EM in Egypt is unknown and it should be noted that this case may be the first case in Egypt reported. 


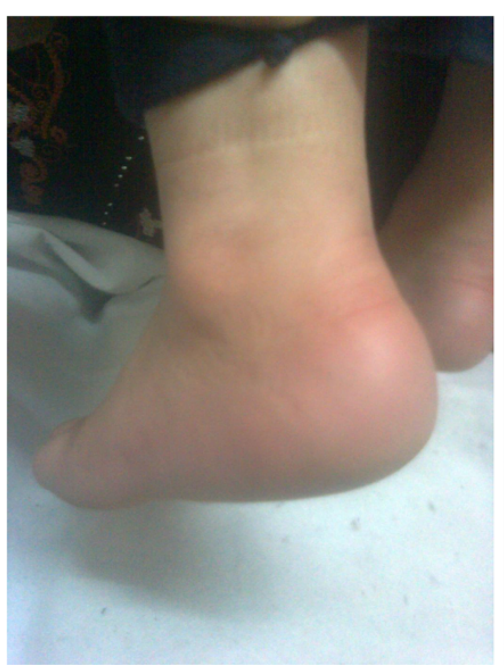

Figure 1 Photography for our case during an episode.

Adult-onset EM has a wide range of age distribution, with most cases occurring in the fifth and sixth decades; in early reports, the median age of onset for early-onset EM was 10 years [5].

After a 37-year analysis of 32 pediatric cases at the Mayo Clinic, Minnesota, researchers concluded that the majority of cases were not inherited and that progress of the disease is variable. No safe or reliable treatment has been established; EM in the pediatric population is associated with substantial morbidity and, sometimes, death [8].

In a Norwegian case series [7], the age range in the primary group (early onset and idiopathic adult onset disease) was 7 to 76 years, and the age range in the secondary group (secondary EM) was 18 to 81 years. In a retrospective review of 168 patients with EM examined at the Mayo Clinic between 1970 and 1994, the median age was 60 years and the age range was 5 to 91 years $[5,8]$.

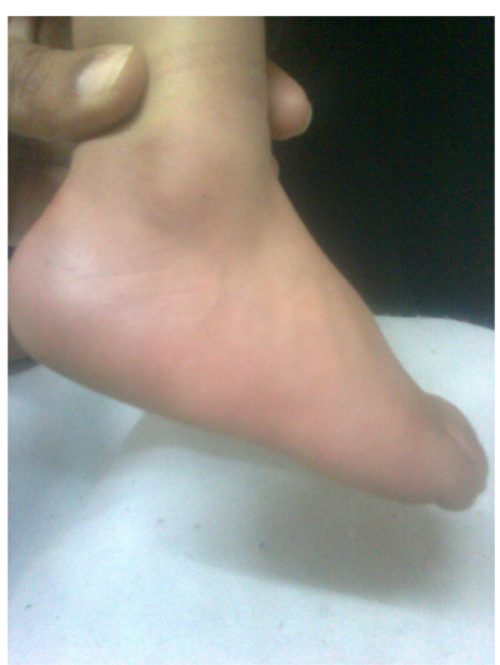

The early age of our child is considered one of the earliest ages for presentation for primary (or idiopathic) EM and this is consistent with the few studies that have reported onset of EM at the age of 3 years or less in some families [9-11].

The Mayo Clinic series showed a male-to-female ratio of $1: 3$, whereas it was $3: 2$ in a review of 60 cases of EM due to myeloproliferative disorders $[5,7,8]$.

As there is a relatively well-documented association between EM and myeloproliferative disorders and systemic mastocytosis, an initial CBC with a differential count as well as bone marrow examination were done which revealed normal values. A normal CBC excluded other hematological disorders including pernicious anemia, essential thrombocythemia, polycythemia vera, myelodysplastic syndrome, and thrombotic and immunologic thrombocytopenic purpuras [1,3]. Hemoglobin electrophoresis
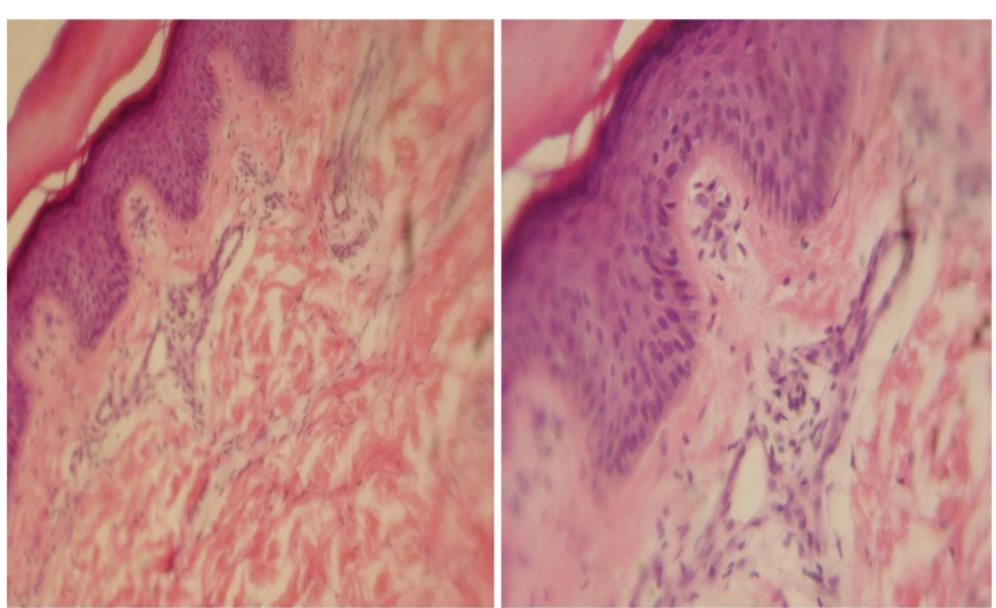

Figure 2 Skin biopsy of the case. 
was done for the exclusion of chronic hemolytic anemias, especially sickle cell anemia, which may elucidate the symptomatology of the child when no abnormality can be detected.

Several authors [5,7] have reported that the onset of symptoms of EM may precede detectable myeloproliferative diseases by several years. So our patient was advised on the benefit of routine follow-up evaluation. EM may be secondary to connective tissue disorders [2], such as rheumatoid arthritis, systemic lupus erythematosus, Sjögren's syndrome and vasculitis, but the clinical presentation and laboratory investigations excluded them because the results of the autoimmune panel were normal. This autoimmune panel included: normal CBC and erythrocyte sedimentation rate, negative rheumatoid factor, and antinuclear, antiSmith and anti-deoxyribonucleic acid (DNA) antibodies.

Some drugs (for example bromocriptine), mushroom ingestion and mercury poisoning $[2,12]$ were reported to cause secondary EM and those were excluded by history and clinical examination.

In our case, there was no X-ray evidence of calcified arteries in his lower limbs. In addition, he had normal blood pressure, normal values of serum cholesterol and triglycerides levels, and normal blood flow of both lower limbs through the Doppler which ruled out EM-like syndromes such as Raynaud's syndrome and Buerger's disease.

Neuropathies (including diabetic neuropathies), multiple sclerosis and spinal cord disease are some of the neurological disorders associated with EM [2]; these were excluded through normal urine analysis, fasting and 2 hours post-prandial blood glucose levels, and normal nerve conduction velocity of both peroneal nerves. The results of a pelvic and abdominal sonography and brain $\mathrm{CT}$, which were done to exclude oncologic causes especially astrocytoma, were normal.

The exact pathological mechanism responsible for this disorder is unknown, but several theories have been proposed to explain its pathophysiology including vasculopathy and/or neuropathy hypotheses. According to the microvascular arteriovenous (AV) hypothesis [13,14], symptoms are caused by tissue hypoxia induced by impaired distribution of skin microvascular blood flow with increased thermoregulatory flow through AV shunts and an inadequate perfusion. Work done by Mork and colleagues [15] supports this hypothesis of increased thermoregulatory flow through $\mathrm{AV}$ shunts during attacks in primary EM, whereas Orstavik and colleagues suggested that EM may be due to hypersensitivity of C-fibers [16].

A prospective study done by Davis et al. [17] suggested that EM is associated with a neuropathy, primarily smallfiber, and a vasculopathy with intermittent increased blood flow and AV shunting. There may be increased local cellular metabolism. However, it is unclear which one is the initiating event or primary abnormality.
Kim et al. [18] reported mutations in the SCN9A gene, which encodes the $\mathrm{Na}_{\mathrm{v}} 1.7$ sodium channel, in patients with primary EM. Cummins et al. [19] demonstrated that these mutations in the $\mathrm{Na}_{\mathrm{v}} 1.7$ channel produce a hyperpolarizing shift in activation and slow deactivation causing the sodium channels to remain open for extended periods of time.

Dib-Hajj et al. [20,21] demonstrated another F1449V mutation in the $\mathrm{Na}_{\mathrm{v}} 1.7$ channel, which also reduces the firing threshold and produces abnormal repetitive firing in sensory neurons in primary EM. Nearly a dozen mutations in $\mathrm{Na}_{\mathrm{v}} 1.7$ have been identified. These mutations have been found to be a cause of familial EM.

Drenth and Waxman [22], Dib-Hajj et al. [23] and Min-Tzu and colleagues [24] reported that mutations in the human SCN9A gene, encoding the $\alpha$-subunit of the voltage-gated sodium channel, $\mathrm{Na}_{\mathrm{v}} 1.7$, were found to be responsible for primary EM. Three missense mutations of the SCN9A gene have recently been identified in Taiwanese patients including a familial (I136V) and two sporadic mutations (I848T, V1316A). V1316A is a novel mutation and has not been characterized yet. Topologically, I136V is located in the DI/S1 segment and both I848T and V1316A are located in S4-S5 linker region of DII and DIII domains, respectively.

Overall, these changes reflect the hyperexcitability of peripheral sensory and sympathetic neurons, which contributes to symptom production in primary EM.

A skin biopsy was done for our child and revealed nonspecific changes as mentioned before; this is consistent with many studies that revealed the same changes $[14,15,25]$.

A universally effective treatment for primary (or idiopathic) EM is still unknown as shown in Table 2. The mainstay of therapy is support and avoidance of trigger factors. Local measures include cooling or elevating the extremity to effectively attenuate or relieve symptoms. Patients should also be counseled about the use of safe cooling options such as fans and air conditioning rather than cold water itself, as ice or immersing an extremity into an icy water bath can lead to skin necrosis and ulceration $[5,8]$.

Treatment with medications such as propranolol, gabapentin, tricyclic antidepressants, sodium nitroprusside, calcium channel blockers and intravenous lidocaine and oral mexiletine had some symptomatic benefits in a few cases and mainly in adults not in children [26-31].

Medications that affect voltage-gated sodium channels show promise, although prostacyclin may provide some benefit and some patients achieved relief with gabapentin or high-dose magnesium [32-34]. Some rare patients with EM may respond well to treatment with carbamazepine especially those with the $\mathrm{Na}_{\mathrm{v}} 1.7$ channel mutations [35].

Recent studies have indicated that it is possible to predict the response of patients with EM to treatment with sodium channel blockers on the basis of atomic-level 
Table 2 Response to earlier medications and other treatments in 32 patients presenting to Mayo Clinic with erythromelalgia [8]

\begin{tabular}{|c|c|c|c|c|}
\hline \multirow[t]{2}{*}{ Drugs used } & \multicolumn{4}{|c|}{ No. of patients, response } \\
\hline & $\mathbf{n}$ & Very helpful & Somewhat helpful & Not helpful \\
\hline Aspirin & 14 & 1 & 2 & 11 \\
\hline NSAIDs (ibuprofen, indomethacin, naproxen) & 14 & 1 & 0 & 13 \\
\hline $\begin{array}{l}\text { Antidepressants (amitriptyline hydrochloride, } \\
\text { venlafaxine, cyproheptadine hydrochloride) }\end{array}$ & 10 & 2 & 2 & 6 \\
\hline Antihistamines (diphenhydramine, cetirizine hydrochloride, cimetidine) & 8 & 0 & 0 & 8 \\
\hline Vasodilators (nitroprusside, nifedipine, diltiazem) & 7 & 1 & 1 & 5 \\
\hline B-blockers (propranolol, atenolol, nadolol) & 6 & 0 & 1 & 5 \\
\hline Narcotics (codeine, morphine, fentanyl) & 6 & 0 & 4 & 2 \\
\hline Gabapentin & 6 & 2 & 0 & 4 \\
\hline Parenteral corticosteroids (oral, intramuscular, intravenous) & 6 & 0 & 1 & 5 \\
\hline Topical corticosteroids & 5 & 0 & 0 & 5 \\
\hline Physical methods (biofeedback, intrathecal pump, TENS unit) & 4 & 1 & 1 & 2 \\
\hline Other anticonvulsants (carbamazepine, phenytoin) & 3 & 0 & 1 & 2 \\
\hline Sympathectomy & 3 & 0 & 1 & 2 \\
\hline Acetaminophen & 3 & 0 & 1 & 2 \\
\hline $\begin{array}{l}\text { Other medications* (doxazosin mesylate, capsaicin, ergotamine tartrate, } \\
\text { mexiletine, clonidine, tetracycline, homeopathic) }\end{array}$ & 7 & 0 & 0 & 7 \\
\hline
\end{tabular}

NSAIDs, nonsteroidal anti-inflammatory drugs; TENS, transcutaneous electrical nerve stimulation.

*Each drug given to one patient and all of them gave a "not helpful" response.

structural modeling [36] raising the possibility that, in the future, it may be possible to genotype patients with EM, and prospectively predict the response to various drugs via pharmacogenomics. We could not perform a genetic study in our case because this was expensive; we depended on the clear history and the clinical picture for the diagnosis of our case. Lastly, genetic study may be more valuable in studies comparing different modalities of therapy.

Antihistamines are often overlooked in the treatment of EM, but these drugs have potent vascular effects and should be considered in difficult cases [37]. Published reports described two remissions with cyproheptadine and three cases with marked improvement using pizotifen, which are antihistamines with proven serotonin antagonist effects at 5-HT2 receptors. In its June 2006 newsletter, The Erythromelalgia Association (TEA) reported a remission that has lasted 10 years with a low dose of cyproheptadine $[37,38]$.

The TEA survey indicates that approximately $40 \%$ of users of antihistamines obtain modest improvement in their EM, whereas $60 \%$ do not obtain improvement. One TEA member reported marked improvement with variable use of desloratadine, chlorpheniramine, and diphenhydramine [37].

Regarding the non-sedating antihistaminic, cetirizine hydrochloride, no improvement was observed in some previous reports $[2,8,37]$ which is in contradiction to our case. This may lead us to think about a new theory of chronic long-standing local allergic reaction which enlightens response to cetirizine in our case in spite of a normal serum IgE titre on the basis of atomic-level structural modeling.

Secondary EM successfully treated with intravenous immunoglobulin plus treatment of the cause in a female patient with seronegative polyarthritis has also been reported [39].

Ulceration, necrosis, and gangrene of affected extremities are possible. Digital necrosis or skin ulceration with secondary infection can lead to amputation. At least one patient had near-fatal hypothermia related to the constant cooling required to control symptoms $[5,8,37]$.

\section{Conclusions}

EM is a rare clinical syndrome of which the etiology, diagnosis and management are controversial. We describe a case of a 34-month-old Egyptian child with primary EM that manifested at an early age. We believe that it is one of the rare cases with early onset of presentation and we believe it is the first Egyptian case report of this kind in the literature. Partial response of this case to cetirizine hydrochloride may grant us a new clue to understanding this mysterious condition.

\section{Consent}

Written informed consent was obtained from the child's father for publication of this case report and any accompanying images. A copy of the written consent is available for review by the Editor-in-Chief of this journal. 


\section{Abbreviations}

AV: Arteriovenous; CBC: Complete blood count; CT: Computed tomography; EM: Erythromelalgia; IgE: Immunoglobulin E; TEA: The Erythromelalgia Association.

\section{Competing interests}

The authors declare they have no competing interests.

\section{Authors' contributions}

SA managed the patient, gathered the data, searched and reviewed the literature. AE searched and reviewed the literature and critically shared in the design and finishing the manuscript. Both authors read and approved the final manuscript.

\section{Acknowledgments}

Our great thanks are given to Professor Nabil G. Mohamed, Head of the Pediatric Department, Minia University, for his kind support to complete this work and for discussion of this case during the staff rounds. We also greatly thank all staff members of the Clinical Pathology Department, Minia University, for their support to finish all the laboratory investigations needed for this case. Lastly, we thank Dr Marian Magdy, Lecturer of Pathology for her efforts to prepare, read and interpret the skin biopsy of our case. This work was funded by the researchers as employees of Minia University.

Received: 10 June 2013 Accepted: 6 January 2014 Published: 25 February 2014

\section{References}

1. Michiels JJ, Drenth JP, Van Genderen PJ: Classification and diagnosis of erythromelalgia and erythermalgia. Int J Dermatol 1995, 34:97-100.

2. Buttaci CJ: Erythromelalgia: a case report and literature review. Pain Medicine 2006, 7:534-538.

3. Bouyahyaoui Y, Meziane M, Hanaae Z, Mikou O, Mernissi FZ, Baba Khouya A, Otmani S, Hida M: Primary familial erythromelalgia. A case report. Arch Pediatr 2013, 20:369-371.

4. Mitchell SW: On a rare vasomotor neurosis of the extremities and on the maladies with which it may be confounded. Am J Med Sci 1878, 76:17-36.

5. Davis MD, O'Fallon WM, Rogers RS, Rooke TW: Natural history of erythromelalgia: presentation and outcome in 168 patients. Arch Dermatol 2000, 136:330-336.

6. Kalgaard OM, Seem E, Kvernebo K: Erythromelalgia: a clinical study of 87 cases. J Intern Med 1997, 242:191-197.

7. Alhadad A, Wollmer P, Svensson A, Eriksson KF: Erythromelalgia: incidence and clinical experience in a single centre in Sweden. Vasa 2012, 41:43-48.

8. Cook-Norris RH, Tollefson MM, Cruz-Inigo AE, Sandroni P, Davis MD, Davis DM: Pediatric erythromelalgia: a retrospective review of 32 cases evaluated at Mayo clinic over a 37-year period. J Am Acad Dermatol 2011, 26:

9. Han C, Rush AM, Dib-Hajj SD, Li S, Xu Z, Wang Y, Tyrrell L, Wang X, Yang Y, Waxman SG: Sporadic onset of erythermalgia: a gain-of-function mutation in $\mathrm{Na}_{\mathrm{v}}$ 1.7. Annals of Neurology 2006, 59:553-558.

10. Estacion M, Dib-Hajj SD, Benke PJ, te Morsche RH, Eastman EM Macala LJ, Drenth JP, Waxman SG: Na 1.7 gain-of-function mutations as a continuum: A1632E displays physiological changes associated with erythromelalgia and paroxysmal extreme pain disorder mutations and produces symptoms of both disorders. J Neurosci 2008, 28:11079-11088.

11. Dib-Hajj SD, Rush AM, Cummins TR, Hisama FM, Novella S, Tyrrell L, Marshall L, Waxman SG: Gain-of-function mutation in $\mathrm{Na}_{\mathrm{v}} 1.7$ in familial erythromelalgia induces bursting of sensory neurons. Brain 2005, 128:1847-1854.

12. Dupont E, Illum F, Olivarius Bde F: Bromocriptine and erythromelalgia-like eruptions. Neurology 1983, 33:670.

13. Kvernebo K: Erythromelalgia - a condition caused by microvascular arteriovenous shunting. Vasa 1998, 51(Suppl):1-39.

14. Mork C, Asker CL, Salerud EG, Kvernebo K: Microvascular arteriovenous shunting is probable pathogenetic mechanism in erythromelalgia. J Invest Dermatol 2000, 114:643-646.

15. Mork C, Kalgaard OM, Kvernebo K: Impaired neurogenic control of skin perfusion in erythromelalgia. J Invest Dermatol 2002, 118:699-703.
16. Orstavik K, Weidner C, Schmidt R, Schmelz M, Hilliges M, Jorum E, Handwerker H, Torebjork E: Pathological C-fibres in patients with a chronic painful condition. Brain 2003, 126:567-578.

17. Davis MD, Sandroni P, Rooke TW, Low PA: Erythromelalgia: vasculopathy, neuropathy or both? Arch Dermatol 2003, 139:1337-1343.

18. Kim MK, Yuk JW, Kim HS, Park KJ, Kim DS: Autonomic dysfunction in SCN9A-associated primary erythromelalgia. Clin Auton Res 2013, 23:105-107.

19. Cummins TR, Dib-Hajj SD, Waxman SG: Electrophysiological properties of mutant $\mathrm{Na}_{\mathrm{v}} 1.7$ sodium channels in a painful inherited neuropathy. J Neurosci 2004, 24:8232-8236.

20. Cheng X, Dib-Hajj SD, Tyrrell L, Te Morsche RH, Drenth JP, Waxman SG Deletion mutation of sodium channel $\mathrm{Na}(\mathrm{V}) 1.7$ in inherited erythromelalgia: enhanced slow inactivation modulates dorsal root ganglion neuron hyperexcitability. Brain 2011, 134:1972-1986.

21. Dib-Hajj SD, Cummins TR, Black JA, Waxman SG: From genes to pain: $\mathrm{Na}_{v} 1.7$ and human pain disorders. Trends Neurosci 2007, 30:555-563.

22. Drenth JP, Waxman SG: Mutations in sodium channel gene SCN9A cause a spectrum of human genetic pain disorders. J Clin Invest 2007, 117:3603-3609.

23. Dib-Hajj SD, Yang Y, Black JA, Waxman SG: The $\mathrm{Na}_{\mathrm{v}} 1.7$ sodium channel: from molecule to man. Nat Rev Neurosci 2012, 14:49-62.

24. Min-Tzu W, Po-Yuan H, Chen-Tung Y, Chih-Cheng C, Ming-Jen L: A novel SCN9A mutation responsible for primary erythromelalgia and is resistant to the treatment of sodium channel blockers. PLoS One 2013, 8:e55212.

25. Skeik N, Rooke TW, Davis MD, Davis DM, Kalsi H, Kurth I, Richardson RC: Severe case and literature review of primary erythromelalgia: novel SCN9A gene mutation. Vasc Med 2012, 17:44-49.

26. Bada JL: Treatment of erythromelalgia with propranolol [letter]. Lancet 1977, 2:412.

27. Ozsoylu S, Caner H, Gökalp A: Successful treatment of erythromelalgia with sodium nitroprusside. J Pediatr 1979, 94:619-621.

28. Nathan A, Rose JB, Guite JW, Hehir D, Milovcich K: Primary erythromelalgia in a child responding to intravenous lidocaine and oral mexiletine treatment. Pediatrics 2005, 115:e504-e507.

29. Cohen JS: High-dose oral magnesium treatment of chronic, intractable erythromelalgia. Ann Pharmacother 2002, 36:255-260.

30. Davis MD, Sandroni P: Lidocaine patch for pain of erythromelalgia: follow-up of 34 patients. Arch Dermatol 2005, 141:1320-1321.

31. Iqbal J, Bhat MI, Charoo BA, Syed WA, Sheikh MA, Bhat IN: Experience with oral mexiletine in primary erythromelalgia in children. Ann Saudi Med 2009, 29:316-318.

32. Legroux-Crespel E, Sassolas B, Guillet G: Treatment of familial erythermalgia with the association of lidocaine and mexiletine. Ann Dermatol Venereol 2003, 130:429-433.

33. Goldberg YP, Price N, Namdari R, Cohen CJ, Lamers MH, Winters C, Price J, Young CE, Verschoof H, Sherrington R, Pimstone SN, Hayden MR: Treatment of $\mathrm{Na}_{\mathrm{v}}$ 1.7-mediated pain in inherited erythromelalgia using a novel sodium channel blocker. Pain 2012, 153:80-85.

34. Davis MD, Rooke T: Erythromelalgia. Current Treatment Options in Cardiovascular Medicine 2006, 8:153-165.

35. Fischer TZ, Gilmore ES, Estacion M, Eastman E, Taylor S, Melanson M Dib-Hajj SD, Waxman SG: A novel $\mathrm{Na}_{\mathrm{v}} 1.7$ mutation producing carbamazepine-responsive erythromelalgia. Ann Neurol 2009, 65:733-741.

36. Yang Y, Dib-Hajj SD, Zhang J, Zhang Y, Tyrrell L, Estacion M, Waxman SG: Structural modeling and mutant cycle analysis predict pharmacoresponsiveness of a $\mathrm{Na}_{\mathrm{v}} 1.7$ mutant channel. Nature Comm 2012, 3:1186.

37. The erythromelalgia association: footSteps: toward progress. Newsletter 2006, 7:6. www.erythromelalgia.org.

38. Sakakibara R, Fukutake T, Kita K: Treatment of erythromelalgia with cyproheptadine. J Auton Nerv Syst 1996, 58:121-122.

39. Moody S, Pacheco S, Butler IJ, Koenig MK: Secondary erythromelalgia successfully treated with intravenous immunoglobulin. J Child Neurol 2012, 27:922-923.

doi:10.1186/1752-1947-8-69

Cite this article as: Al-Minshawy and El-Mazary: An Egyptian child with erythromelalgia responding to a new line of treatment: a case report and review of the literature. Journal of Medical Case Reports 2014 8:69. 